\title{
NOTE
}

\section{FACTOR-SUPPLY RESPONSES AND THE GROSS-SUBSTITUTE SYSTEM}

\author{
Tapan BISWAS \\ University of Hull, Hull HU6 7RX, U.K. \\ Communicated by F.W. Roush \\ Received 29 October 1984
}

\begin{abstract}
Although the inputs are not normally gross-substitutes in their demand, the Jacobian matrix of all excess demand functions, corresponding to both outputs and inputs, may satisfy the grosssubstitute requirements if, apart from the final goods being gross-substitutes in their demand, all inputs are substitutes in supply.
\end{abstract}

Key words: Gross-substitute system; Metzler-Mosak matrix; net input transformation curve.

\section{Introduction}

Gross-substitute systems play a vital role in many important results in general equilibrium theory. First introduced by Hicks (1937) and then generalised by Morishima (1970), the assumption that commodities are gross-substitutes has pervaded the literature on the uniqueness of competitive equilibrium, comparative static analysis (Hicksian Laws, Le Chatelier-Samuelson Principle), stability analysis and a large number of other related areas (e.g. see Arrow and Hahn, 1971). The logical consistency of this assumption was questioned in Rader (1968) in the context of a production economy, by showing that inputs are not normally gross-substitutes in their demand. This was confirmed by Sakai (1974) in a more general set up. The thrust of this result is that the gross-substitute systems, although consistent with an exchange economy, cannot be used to characterise a production economy with fixed supply of inputs. An attempt was made in Kusumoto (1976) to solve the problem by imposing exogenous constraints on the demand for inputs. However the signpattern of the matrix of constraint-coefficients does not seem to have any economic interpretation and seems to provide a way out of Rader's problem only in a mechanical way. In this note, we observe that although inputs are not normally gross-substitutes in their demand, the excess demand functions for inputs may satisfy the gross-substitute property (the Jacobian matrix having positive offdiagonal elements) only if in the system all the inputs are substitutes in supply. If 
all the inputs are substitutes in supply, then the net input transformation curve must satisfy some restrictions which have been examined here. In a general equilibrium set up with production, the supply of outputs is responsive to changes in both output and input prices. It is therefore appropriate that we should consider the supply of inputs to be responsive to price changes, i.e. in line with the tradition of general equilibrium theory, the production of inputs should be explicitly incorporated into the structure of the economy.

\section{Framework of analysis and results}

Write the production function of the output, $y$, produced by the firm as

$$
y=f\left(x_{1}, \ldots, x_{n}\right), \quad f_{i j}>0, \text { for } i \neq j \text { and } f_{i i}<0 .
$$

We also assume $f(x)$ to be strictly concave (to satisfy second-order condition for profit maximisation) so that the Hessian Matrix $\left[f_{i j}\right]$ is a negative definite matrix. The individual producer maximises his profit, $p \cdot f-w \cdot x$. The first-order condition for maximisation yields, $p \cdot f_{i}=w_{i}$. Taking differentials, we obtain $\delta x=p^{-1}\left[f_{i j}\right]^{-1} \delta w$. Since, $f_{i j}>0$ for $i \neq j, f_{i i}<0$ and $\left[f_{i j}\right]$ is a negative definite matrix, $\delta x_{i} / \delta w_{j}<0$, for $i, j=1,2, \ldots, n$. Let $X_{i}$ stand for the aggregate demand for the $i$ th input. It follows that the matrix of input demand responses $\left[\delta X_{i} / \delta w_{j}\right]$ is a negative matrix and if input supply is fixed, then the excess demand functions for inputs do not satisfy the gross-substitute requirements. This is the basic result in Rader (1968). Consider the production of inputs and let the net input transformation curve for the economy be given by,

$$
T\left(\hat{X}_{1}, \ldots, \hat{X}_{n}\right)=0, \quad T_{i}>0 .
$$

As usual, the net input transformation curve is assumed to be strictly concave, i.e. if $T\left(\hat{X}^{1}\right)=T\left(\hat{X}^{2}\right)=0$ and $\hat{X}^{3}=\alpha \hat{X}^{1}+(1-\alpha) \hat{X}^{2}, 0<\alpha<1$, then $T\left(\hat{X}^{3}\right)<0 . \hat{X}_{i}$ denotes the aggregate supply of the $i$ th input. Under competition, production is efficient which implies:

$$
T_{i} / T_{j}=w_{i} / w_{j} ; \quad \hat{X}_{i}=\hat{X}_{i}\left(w_{1}, \ldots, w_{n}\right) .
$$

Since the supply function is homogeneous of degree 0 in input prices,

$$
\sum_{j} w_{j}\left(\delta \hat{X}_{i} / \delta w_{j}\right)=0
$$

Theorem 1. $\hat{X}_{i}$ and $\hat{X}_{j}$ are substitutes in supply if $\delta \hat{X}_{i} / \delta w_{j}=\delta \hat{X}_{j} / \delta w_{i}<0$, and if all inputs are substitutes in supply, then the matrix $\left[\delta \hat{X}_{i} / \delta w_{j}\right]$ has positive diagonal and negative off-diagonal elements.

No proof is necessary. For detailed discussions on substitutes and complements in supply (production) see Russell and Wilkinson (1979, ch. 7) which also provides proofs for the results $\delta \hat{X}_{i} / \delta w_{j}=\delta \hat{X}_{j} / \delta w_{i}$ and $\delta \hat{X}_{i} / \delta w_{i}>0$. 
Theorem 2. The Jacobian matrix of excess demand functions for inputs will satisfy the gross-substitute requirements if and only if all the inputs are substitutes in supply and $\delta \hat{X}_{i} / \delta w_{j}<\delta X_{i} / \delta w_{j}, i \neq j$.

Proof. Let $E_{i}$ denote the excess demand for the $i$ th input. By definition, $\delta E_{i} / \delta w_{j}=$ $\delta X_{i} / \delta w_{j}-\delta \hat{X}_{i} / \delta w_{j}$. Since, $\delta X_{i} / \delta w_{i}<0$ and $\delta \hat{X}_{i} / \delta w_{i}>0, \delta E_{i} / \delta w_{i}<0$. As $\delta X_{i} / \delta w_{j}<$ 0 for $i \neq j, \delta E_{i} / \delta w_{j}>0$ if and only if $\delta \hat{X}_{i} / \delta w_{j}<\delta X_{i} / \delta w_{j}<0$ for $i \neq j$.

The Jacobian of the excess demand functions may have a gross-substiute signpattern only if all the inputs are substitutes in supply. One is naturally interested in the nature of restrictions on the net input transformation curve, implied by this assumption. For an arbitrary choice of $n$, equation (1) may be written as

$$
\hat{X}_{n}=\theta^{n}\left(\hat{X}_{1}, \hat{X}_{2}, \ldots, \hat{X}_{n-1}\right) \text {. }
$$

The assumption of a concave net input transformation curve implies that the Jacobian matrix of $\theta^{n}$ is negative definite. In a competitive economy, $w_{i} / w_{n}=-\theta_{i}^{n}$, $i=1,2, \ldots, n-1$. Assuming $w_{n}=1$,

$$
\left[-\theta_{i j}^{n}\right]\left(\delta \hat{X}_{1}, \delta \hat{X}_{2}, \ldots, \delta \hat{X}_{n-1}\right)=\left(\delta w_{1}, \delta w_{2}, \ldots, \delta w_{n-1}\right) .
$$

Theorem 3. If all inputs are substitutes in supply, then $\left[\theta_{i j}^{n}\right]$ is $a(n-1) \times(n-1)$ negative matrix for any arbitrary choice of $n$.

Proof. From equation (6), $\left[\delta \hat{X}_{i} / \delta w_{j}\right]=\left[-\theta_{i j}^{n}\right]^{-1}, i, j=1,2, \ldots, n-1$. If all inputs are substitutes in supply, $\left[-\theta_{i j}^{n}\right]^{-1}$ is a matrix with positive diagonal and negative offdiagonal elements. Since, $\delta \hat{X}_{i} / \delta w_{n}<0$, using equation (4) we establish that $\left[-\theta_{i j}^{n}\right]^{-1}$ is a quasi-dominant diagonal (positive definite) matrix. As in Rader (1968), by using the Metzler-Mosak theorem, $\left[-\theta_{i j}^{n}\right]$ is a positive matrix. Therefore $\left[\theta_{i j}^{n}\right]$ is a negative matrix. Since all inputs are substitutes, $\delta \hat{X}_{i} / \delta w_{j}<0$ for $i \neq j, i, j=1,2, \ldots, n$ which requires $\left[\theta_{i j}^{n}\right], i, j=1,2, \ldots, n-1$, to be a negative matrix for any arbitrary $n$.

Theorem 4. Consider a production economy where (i) the final goods $\left(Y_{i}\right)$ are grosssubstitutes in their demand, (ii) the individual producers are maximising their profit, $p_{j} \cdot y_{j}-\sum w_{i} \cdot x_{i}$ (no joint production of final goods) and $y_{j}=f_{j}\left(x_{1}, x_{2}, \ldots, x_{n}\right.$ ) is strictly concave, (iii) all inputs are substitutes in supply and satisfy the conditions in Theorem 2. The Jacobian matrix of the excess demand functions, $(E(Y), E(X))=$ $G(p, w)$, will have positive off-diagonal elements. $(E(Y)$ and $E(X)$ are the vectors of the excess demand functions for the final goods $\left(Y_{j}\right)$ and inputs $\left(X_{i}\right)$ respectively.)

Proof. Let there be $m$ final goods and $n$ inputs. By $J$ denote the Jacobian of the mapping $G$. The matrix $J$ may be partitioned as follows:

$$
J=\left[\begin{array}{ll}
A_{m \times m} & B_{m \times n} \\
C_{n \times m} & D_{n \times n}
\end{array}\right] .
$$


Since there is no joint production in final foods, $\delta Y_{i} / \delta p_{j}=0, i \neq j$, and because $Y_{i}$ and $Y_{j}$ are gross-substitutes in demand, $A$ has positive off-diagonal elements. Since $f_{i}(x)$ is strictly concave $\delta \hat{Y}_{i} / \delta w_{j}<0$ and $\delta Y_{i} / \delta w_{j}=0$ (demand for final goods is independent of input prices). Therefore, $B$ is a positive matrix. Again the strict concavity of $f_{i}(x)$ together with profit maximisation imply $\delta X_{i} / \delta p_{j}>0$ and $\delta \hat{X}_{i} / \delta p_{j}=0$. Hence, $C$ is a positive matrix. By condition (iii), $D$ has positive off-diagonal elements. Therefore, $J$ has positive off-diagonal elements.

We have shown that if inputs are producible and are substitutes in supply, the Jacobian matrix for the excess demand functions of the economy as a whole may have the sign-pattern of a gross-substitute matrix although inputs are not normally gross-substitutes in their demand.

All inputs may be locally substitutes in supply, even if all of them are not producible. In the context of a non-linear input-output framework for input prodution, let $\bar{X}=\left(\bar{X}_{1}, \bar{X}_{2}\right)$ be the initial composition of the vector of inputs. The net production of inputs is given by,

$$
\begin{aligned}
& \hat{X}_{1}=F\left(X_{1}, X_{2}\right), \\
& \hat{X}_{2}=\bar{X}_{2}-X_{2}, \\
& 0 \leq X_{1} \leq \bar{X}_{1} ; \quad 0 \leq X_{2} \leq \bar{X}_{2} .
\end{aligned}
$$

The set which includes all configurations like $\hat{X}=\left(\hat{X}_{1}, \hat{X}_{2}\right)$ is called the net input availability set. The net input transformation curve is the set $T=(\hat{x} \in \hat{X}$ and $x>\hat{x} \Rightarrow$ $x \notin \hat{X}$ ). The set $T$ when diagrammatically represented as a curve in $E^{n}$ may have regions where all net inputs are substitutable, although the elements in $X_{2}$ are not producible inputs. This is possible, of course, where the net available inputs of the non-producible type are less than their initial supply. As an illustration, let us define $x_{1}$ and $x_{2}$ as machines and labour, respectively. Labour is fixed in supply whereas machines are producible.

$$
\begin{aligned}
& \hat{x}_{1}=f\left(x_{1}, x_{2}\right) ; \\
& \quad f \text { is increasing and strictly concave in its arguments, } \\
& \hat{x}_{2}=\bar{x}_{2}-x_{2}, \\
& 0 \leq x_{1} \leq \bar{x}_{1} ; \quad 0 \leq x_{2} \leq \bar{x}_{2} .
\end{aligned}
$$

The function $\hat{x}_{1}=f\left(\bar{x}_{1}, \bar{x}_{2}-\hat{x}_{2}\right)$ defines the net input transportation function in this non-linear input-output technology. if $\hat{x}_{2}<\bar{x}_{2}$, then $\delta \hat{x}_{1} / \delta \hat{x}_{2}<0, \delta^{2} \hat{x}_{1} / \delta \hat{x}_{2}^{2}=f_{22}<0$, which imply that $\hat{x}_{1}$ and $\hat{x}_{2}$ are substitutes in supply. Fig. 1 illustrates the transformation curve. At $\hat{x}$, both labour and machines are substitutes in supply. 


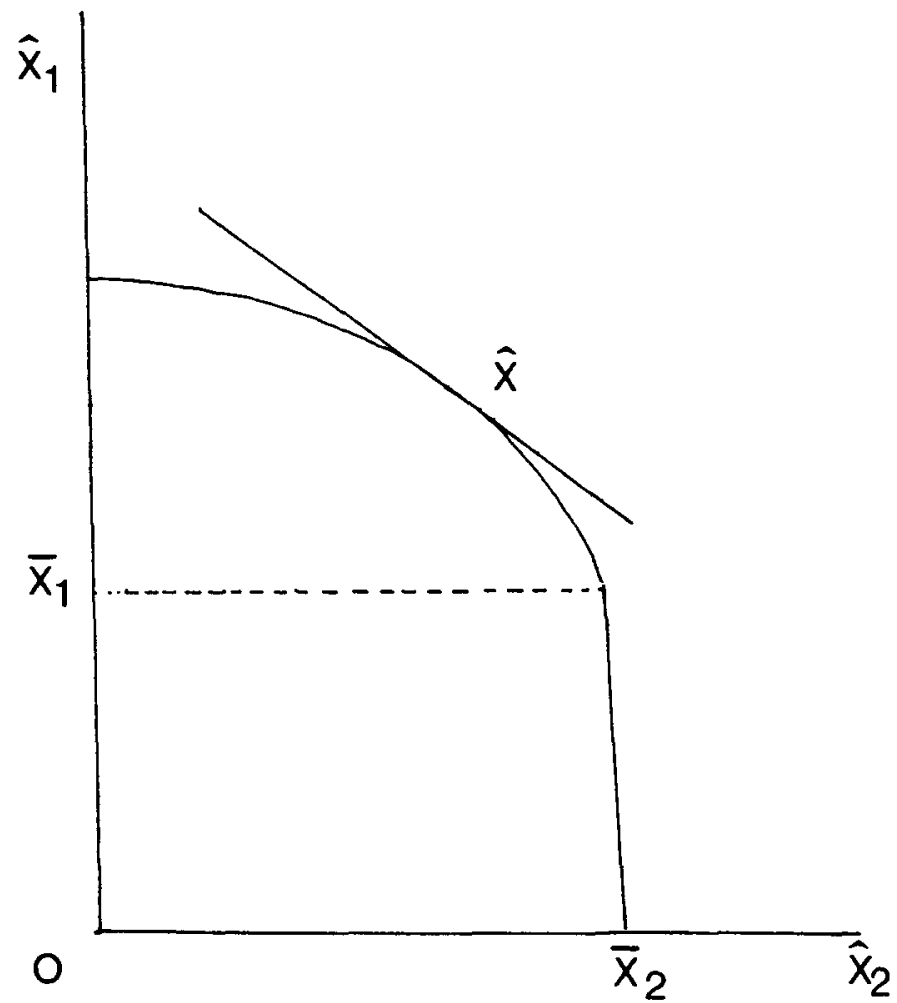

Fig. 1.

\section{Conclusion}

Let us briefly summarise the importance of what we have discussed here. As explained earlier, a large bulk of results in general equilibrium theory depends on the assumption of gross substitutability. Rader (1968) dealt a severe blow to the validity of these results by pointing out that such an assumption, although plausible in an exchange economy, is logically inconsistent with a production economy where the supply of inputs is fixed. We have argued that if some of the inputs are producible, then there is no logical inconsistency in assuming a gross-substitute sign-pattern for the Jacobian of the excess demand functions, even in a production economy.

\section{References}

K.J. Arrow and F.H. Hahn, General Competitive Analysis (Oliver and Boyd, Edinburgh, 1971).

J.R. Hicks, Value and Capital (Clarendon Press, Oxford, 1937).

S-I. Kusumoto, Extensions of the Le Chatelier-Samuelson principle and their application to analytical economics, Econometrica 44 (1976) 509-535.

M. Morishima, A generalisation of the gross-substitute system, Rev. Econom. Studies 37 (1970) 177-186.

T. Rader, Normally factor inputs are never gross substitutes, Journal of Political Economy 76 (1968) 38-43.

R. Russell and M. Wilkinson, Microeconomics (John Wiley, New York, 1979).

Y. Sakai, Substitution and expansion effects in production theory: the case of joint production, J. Econom. Theory 9 (1974) 255-274. 